\title{
Numerical simulation of an experimental analogue of a planetary magnetosphere
}

\author{
Andy Sha Liao ${ }^{\mathrm{a}, *}$, Shule Li $^{\mathrm{b}}$, Patrick Hartigan ${ }^{\mathrm{a}}$, Peter Graham ${ }^{\mathrm{c}}$, Gennady Fiksel ${ }^{\mathrm{b}}$, Adam Frank $^{\mathrm{b}}$, John Foster $^{\mathrm{c}}$, Carolyn Kuranz $^{\mathrm{d}}$ \\ ${ }^{a}$ Rice University, 6100 Main St., Houston, TX 77005 \\ ${ }^{b}$ University of Rochester, 500 Joseph C. Wilson Blvd., Rochester, NY 14627 \\ ${ }^{c}$ Awe PLC, Aldermaston, Reading, Berkshire RG7 4PR, United Kingdom \\ ${ }^{d}$ University of Michigan, 500 S. State St., Ann Arbor, MI 48109
}

\begin{abstract}
Recent improvements to the Omega Laser Facility's magneto-inertial fusion electrical discharge system (MIFEDS) have made it possible to generate strong enough magnetic fields in the laboratory to begin to address the physics of magnetized astrophysical flows. Here, we adapt the MHD code AstroBEAR to create 2D numerical models of an experimental analogue of a planetary magnetosphere. We track the secular evolution of the magnetosphere analogue and we show that the magnetospheric components such as the magnetopause, magnetosheath, and bow shock, should all be observable in experimental optical band thermal bremsstrahlung emissivity maps, assuming equilibrium charge state distributions of the plasma. When the magnetosphere analogue nears the steady state, the mid-plane altitude of the magnetopause from the wire surface scales as the one-half power of the ratio of the magnetic pressure at the surface of the free wire to the ram pressure of an unobstructed wind; the mid-plane thickness of the magnetosheath is directly related to the radius of the magnetopause. This behavior conforms to Chapman and Ferraro's theory of planetary magnetospheres. Although the radial dependence of the magnetic field strength differs between the case of a current-carrying wire and a typical planetary object, the major morphological features that develop when a supersonic flow passes either system are identical. Hence, this experimental concept is an attractive one for studying the dynamics of planetary magnetospheres in a controlled environment.
\end{abstract}

Keywords: Omega Laser Facility, MIFEDS, Laser astrophysics, Planetary magnetospheres, MHD, Methods: Numerical

\section{Introduction}

The magnetic force, and hence magnetic pressure, contributes significantly to the dynamics that govern many astrophysical phenomena including accretion columns feeding young stars [1], starspots [2], flows of gas in stellar coronae [3, 4, 5, 6], and the space environment near magnetized planets [7, 8, 9, 10]. The magnetic field plays a key role in these objects by constraining the cross-field motion of the plasma inside the magnetized region, and as a medium of energy storage and transport. In the case of magnetized accretion columns, simulations show that when the magnetic pressure is a significant contributor to the total pressure of the gas within the column, splashing of the accreting material as it impacts the stellar surface was greatly reduced from that observed in the case of a non-magnetized accretion column [1]. On stellar photospheres, including that of the Sun, the increased magnetic pressure in the vicinity of bunched magnetic flux tubes can impede the normal transport of energy from the stellar interior. This produces cooler and darker regions on the stars' surface observable as starspots [2]. Additionally, observations and MHD simulations demonstrate that stellar coronae are given their global appearance by the stellar magnetic field that binds and directs the flows of plasma. Sudden relaxations of the magnetic constraints, particularly by magnetic reconnections can produce

\footnotetext{
${ }^{*}$ Corresponding author.

Email address: Andy.Liao@rice.edu (Andy Sha Liao)
}

energetic events including flares and coronal mass ejections (CMEs) [3, 4, 6]. Finally, strong magnetic fields generated by a planetary dynamo deflect the solar wind high above the planet's surface. The interaction between the planetary magnetic field and the solar wind produces the structures of the planet's magnetosphere [7, 8].

Chapman and Ferraro [7, 11, 12, 13, 14, 15] developed the first modern theory of planetary magnetospheres in the 1930's. In their model, the edge of the solar wind forms a conducting surface in its approach towards a magnetized planet. When the wind plasma deflects around the planet's magnetic field, the magnetic field anchored to the planetary dynamo reacts through a compression on its windward side, and a rarefaction into a long tail on its leeward side. The solar wind compresses the planetary field by the same amount as would occur from the superposition of an image magnetic dipole upon the original planetary field, where the source of the image dipole is located upstream from the wind stagnation point by a distance equal to that of the downstream separation of the planet from the stagnation point. As the wind deflects around the planet and its magnetic field, the wind's inner edge settles where the magnetic pressure and the wind's ram pressure balances, and forms the planet's magnetopause. When the supersonic solar wind encounters a magnetized planet, the magnetopause acts as the effective surface of the planet-as-obstacle. A bow shock [16, 17, 18, 19] forms at the location as expected when the supersonic wind encounters a non-magnetized planet with a ra- 
dius equal to that of the magnetopause around a magnetized planet.

However, real magnetospheres are neither static, nor are they as simple as modeled in Chapman and Ferraro's theory. For example, terrestrial magnetic storms arise due to the time variations in the direction of the magnetic field embedded in the solar wind stream. Variations in the solar wind density and velocity also affect the magnetosphere. Given the complexities of real magnetospheres, it is desirable to develop a platform to study the phenomena in a controlled laboratory environment. Any laser experimental platform that replicates the basic physics of planetary magnetospheres may be extended to explore more complex magnetospheric phenomona such as storms. To date, laser experiments have not successfully reproduced the global properties of a magnetosphere because highly magnetized supersonic flows are a new frontier in high-energy-density laboratory techniques [20].

In this paper, we discuss a new laser experimental concept to reproduce the behaviors and structures seen in a steady-state planetary magnetosphere. We describe the experimental design and our models in Section 2 and in Section 3 we show the results of our numerical simulations. In Section 4 we discuss the overall feasibility of realizing the experiment and compare our results with predictions from the Chapman-Ferraro theory.

\section{Model}

\subsection{Wire concept}

A relatively simple way to test the Chapman-Ferraro law is to employ a straight current-carrying wire as the analogue to the planetary magnetic field. The main disadvantages that arise from this scheme are geometric in origin: The wire-as-obstaclein-flow is cylindrical, not spherical like a planet the real solar wind encounters. The magnetic field that is generated by a straight wire is also cylindrical, and its strength falls off with distance $R$ from the wire axis with $R^{-1}$, again deviating from the astrophysical scenario of a planetary dipole field that falls off with distance with $R^{-3}$. In spite of the differences between our scheme and the astrophysical scenario, the Chapman-Ferraro law holds regardless of the particular geometry of the magnetic field, and remains testable in our model as long as the magnetic field is strong enough to deflect the wind at a visibly higher altitude from the wire surface than what is expected when the wind encounters a nonmagnetized wire.

\subsection{Wire simulations}

Our numerical models were completed using AstroBEAR, an AMR radiation and magneto- hydrodynamics code developed at the University of Rochester [21]. In our AstroBEAR simulations, a 2D grid of 501 by 501 cells, or in physical dimensions a square of $2.5 \mathrm{~mm}$ was initialized:

First, an infinitely-long wire object of $250 \mu \mathrm{m}$ radius was inserted into the center of the grid square in cross-section. An ambient medium took the place of the remaining space on the grid.
A magnetic field was imposed, ad-hoc, in the space surrounding the wire to simulate a current flowing through the wire. The vector potential of the imposed magnetic field was

$$
A=(20 \mathrm{~T})(250 \mu \mathrm{m}) \ln \left(\frac{R}{250 \mu \mathrm{m}}\right) \hat{z}
$$

where $R$ for $R>250 \mu \mathrm{m}$ is the radial distance from the wire axis and the grid spans the xy plane. Finally, a wind object was initialized to supply a constant, uniform flux of material from one edge of the grid during runtime.

The ratio of initial densities of ambient medium, wind and wire was maintained to be $3: 40: 3 \times 10^{5}$ throughout this series of simulations even as the density and velocity of the wind was changed from run to run in order to explore the parameter space. The temperature of the wind and ambient medium was maintained across all simulations to be $300 \mathrm{~K}$; the temperature of the wire was taken to be $3 \mathrm{mK}$ to maintain pressure equilibrium between the wire and ambient medium.

The equation of state of all components was taken to be that of the ideal gas and the MHD calculations were nonresistive for neutral plasmas. Evolution was tracked up to 5.3 wire-crossing times, i.e. the time it takes for the wind at onset to cross 500 $\mu \mathrm{m}$ down range, the wire diameter. Snapshots for further processing were taken at 3.9 wire-crossing times.

\subsection{Post-processing}

In order to simulate the data produced by the Gated Optical Imager (GOI) [22] at the Omega Laser Facility, maps of free-free emissivity in the optical band between $400 \mathrm{~nm}$ and $700 \mathrm{~nm}$ wavelengths were created using the formula for thermal bremsstrahlung emissivity [23],

$$
\begin{aligned}
\epsilon_{\mathrm{ff}} & =Q_{z}(T) \times n_{A r}^{2} \times 6.8 \times 10^{-38} T^{-1 / 2} \times \\
& \times\left[\frac{k T}{h}\right] \int_{4.286}^{7.500} \exp \left(-\frac{h v_{14}}{k T}\right) \mathrm{d} v_{14}
\end{aligned}
$$

where $v_{14}$ is the frequency in $10^{14} \mathrm{~Hz}$ and $Q_{z}(T)$ is

$$
Q_{z}(T)=\sum_{i} X_{i}(T)^{2} Z_{i}^{3}
$$

for $Z$ charge and $X$ fraction of argon nuclei in charge state $i$, taken from IAEA-FLYCHK atomic database [24] for equilibrium charge state distributions.

An example map, Fig. 1 shows the normalized optical freefree emissivity in logarithmic scale. This map was created from simulation data taken at the snapshot time of 3.9 wire-crossing times. At this time, the wind coming into the grid from the left side had deflected around the wire, and in the course of its navigation around the obstacle components of the magnetosphere including magnetopause, magnetosheath and bow shock became amenable to measurement by visual inspection. The bow shock had by this time stabilized its position and the distortion of the wire due to compression by the wind had yet to occur to an appreciable degree. 


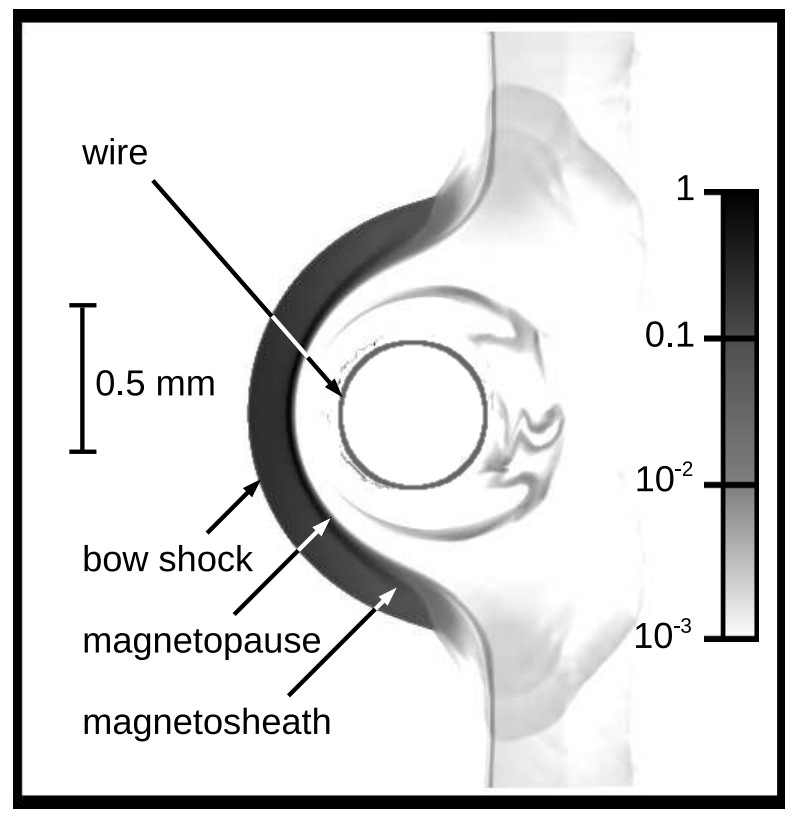

Figure 1: Normalized logarithmic-scaled free-free emissivity map was created from a snapshot at 3.9 wire-crossing times for a simulation with a magnetized wire. Because of the magnetic field surrounding the wire, the wind was deflected roughly $100 \mu \mathrm{m}$ above wire's surface marked by the circle. The emission source region, having detached from the wire, forms the magnetosheath. The magnetosheath is bound by a bow shock on its windward side and a magnetopause on its leeward side. The arrangement seen here replicates the structure of a planetary magnetosphere.

\section{Results}

Fig. 2 shows the measurements, taken on the horizontal midplane of the grid, of the altitude from the wire surface of the magnetopause and the bow shock. Each simulation contributed to one set of measurements for the magnetopause and bow shock; each measurement was taken from a snapshot image like that shown in the example in Fig. 1. at 3.9 wire-crossing times. The measurements for each run were plotted against $1 / \sigma$, where $\sigma=\left(8 \pi \rho v^{2}\right) /\left(B_{0}^{2}\right)$ is the ratio of the ram pressure of the unobstructed wind to the magnetic pressure at the surface of the free wire, pertinent to that run in particular. The $\mathrm{x}$-axis of $1 / \sigma$ values was explored only by adjusting the wind ram pressure, the magnetic field was unchanged from run to run.

In addition to measured results of simulated emissivity maps, the theoretical relations between the magnetopause altitude and $1 / \sigma$, as well as a semiempirical relation between the bow shock altitude and $1 / \sigma$ were also plotted on the same figure for reference. The relation for the theoretical magnetopause altitude is

$$
R_{\text {magnetopause }}=(250 \mu \mathrm{m}) \sigma^{-1 / 2}
$$

and the relation for the bow shock altitude was adapted from the results of Zhuang and Russell [19] to be

$$
R_{\text {bow }}=(1 / 2)\left(R_{0}+R_{\text {magnetopause }}\right)+R_{\text {magnetopause }}
$$

where $R_{0}=250 \mu \mathrm{m}$ is the wire radius.

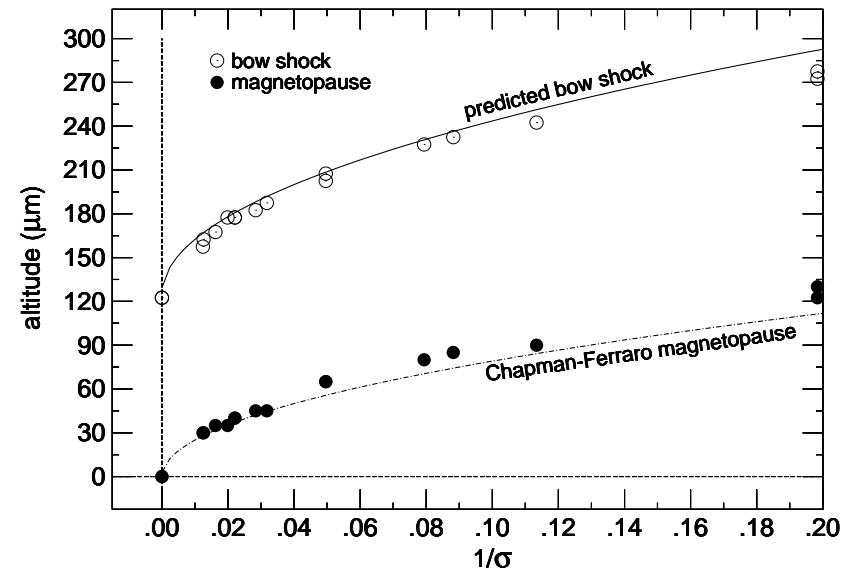

Figure 2: Simulated bow shock and magnetopause altitudes with input parameters spanning a range of $1 / \sigma$ were plotted against fiducial curves for those features. Whereas the model results were derived by inspecting the inner and outer boundaries of the magnetosheath in free-free emissivity map snapshots taken at 3.9 wire-crossing times, the fiducial curves were obtained from adapting static planetary theories to the cylindrical geometry of the models. The GOI, with a resolution of $30 \mu \mathrm{m}$, should be able to resolve the offset from the hydrodynamic case of either the bow shock or magnetopause altitude by even a weak magnetic field.

\section{Discussion and summary}

Given Eq. (2) and (3), the free-free emissivity depends on the square of density, on the square-root of temperature and on a charge density factor $Q_{z}$ that strongly depends on temperature. Consequently, in every run of the simulation, the free-free emissivity map showed that the emission source region was strongly localized to the space immediately leeward of the bow shock due to the postshock compression, thermalization and ionization of the wind that occurs there. In the example, Fig. 1 1 showed a detachment of the source region from the physical boundary of the wire. This is a consequence of the magnetization of the wire that raised its effective boundary to the location where the pressure from the magnetic field balanced the ram pressure of the wind, i.e. the magnetopause. Following the terminology of planetary magnetospheres, the emission source region, being sandwiched between the bow shock and magnetopause was identified as the magnetosheath. As the magnetosheath developed on the windward side of the wire, a magnetic structure analogous with the magnetotail took root at mid-latitudes of the wire on the leeward side. However, the magnetotail remained purely a magnetic structure; it was entirely encapsulated within the magnetopause cavity and the depletion of hot gas within this region precluded it from being a significant source of emissions.

The Chapman-Ferraro magnetopause standoff distance defines the location from the surface of the magnetized object along the midplane where the magnetic pressure and wind ram pressure balances. Given a power-law relation between the magnetic field strength and the distance from the object surface $R$, the magnetopause altitude depends on a power of $1 / \sigma$. In the case of a dipole field produced by real planetary dynamos, the magnetic field strength depends on $R^{-3}$ and the magnetopause 
altitude depends on $(1 / \sigma)^{1 / 6}$; in the case of a cylindrical field produced by a straight wire, the magnetic field strength depends on $R^{-1}$ and the magnetopause altitude depends on $(1 / \sigma)^{1 / 2}$, as given by Eq. (4). In Fig. 2 the measured magnetopause altitude closely followed the Chapman-Ferraro relation for cylindrical fields. Furthermore, the observed bow shock positions closely followed the positions predicted in Eq. (5) for a bow shock arising from a supersonic wind encountering a cylindrical obstacle with radius equal to that of the magnetopause. Hence, both the theoretical and numerical results suggested that differences may be readily observable between runs of the experiment with and without magnetization of the wire. For example, when $1 / \sigma$ was 0.07 , the bow shock position was displaced nearly $100 \mu \mathrm{m}$ from that seen in the hydrodynamic case, and the magnetopause detached from the wire surface to an altitude of over $60 \mu \mathrm{m}$. Given a diagnostic resolution of $30 \mu \mathrm{m}$, the deflection of the wind from even a relatively weak magnetic field should be detectable.

However, a small discrepancy remained between the data and the theoretical curves in the sense that the the bow shock data lie $5 \mu \mathrm{m}$ to $15 \mu \mathrm{m}$ below the theoretical curve everywhere along the $\mathrm{x}$-axis. Similarily the magnetopause data are $5 \mu \mathrm{m}$ to $10 \mu \mathrm{m}$ above the analytic curve, especially at higher $1 / \sigma$. These discrepancies likely arose due to the timing of the snapshots. The theoretical curves in Fig. 2 hold for the steady-state magnetosphere, while our model data were taken at 3.9 wire- crossing times, before the steady state could be reached.

Fig. 3 shows the secular evolution of a typical model magnetosphere. At times earlier than 2.7 wire-crossing times, the bow shock and magnetopause have yet to form. The data that is labeled as the bow shock show instead the edge of the reverse shock that formed from the interaction of the wind with the ambient medium; the data that is labeled as the magnetopause altitude show instead the location of the contact discontinuity (CD) between the wind and the ambient medium. At times just before 2.7 wire-crossing times, the $\mathrm{CD}$ decelerates as it comes under the gradually increasing influence of the magnetic field; the reverse shock maintains its constant speed of approach, and the shock thickness decreases as a consequence. At 2.7 wirecrossing times, the reverse shock has caught up with the decelerating $\mathrm{CD}$; when the reverse shock reflects from the $\mathrm{CD}$, it becomes the bows shock, and the CD forms the magnetopause. Past this inflection point, the bow shock altitude rises asymptotically towards its steady-state value, while the magnetopause altitude oscillates around its analytic value. The oscillation frequency is higher when $1 / \sigma$ is smaller, and hence, the discrepancy between the theoretical and measured magnetopause altitude in Fig. 2 is more pronounced for runs with higher $1 / \sigma$ because the upward phase of the initial oscillation cycle extends past the time when the snapshot is taken, i.e. 3.9 wire-crossing times. In summary, the secular evolution of the magnetosphere is mostly complete at 3.9 wire-crossing times, and taking our data at a later time would have been unadvisable because as the magnetosphere asymptotically develops toward the steady state, the wire is irreversibly deforming under pressure from the wind.

Taken together, the simulation results and emissivity maps

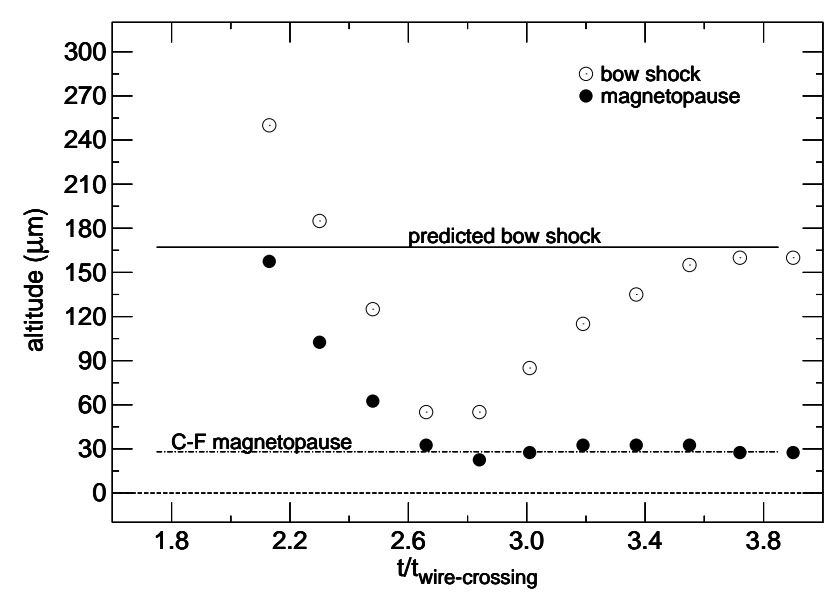

Figure 3: The altitudes of the magnetopause and the bow shock were plotted as a function of time to show the secular evolution of a model magnetosphere from the initial approach towards the obstacle by the $\mathrm{CD}$ up to 3.9 wire-crossing times. At 3.9 wire-crossing times, the magnetopause and bow shock altitudes have mostly completed their evolution towards their steady-state values indicated by the horizontal lines.

showed that the Chapman-Ferraro law can be validated in the HEDP experimental concept modeled here, in spite of a few simplifying assumptions. We conclude that even a weak magnetic field should have an observable effect on the bow shock position as measured with existing diagnostic imagers.

\section{Acknowledgements}

This work was made possible through DOE funding via the NLUF program DE-FOA-0000681, and by the NSF Cyberinfrastructure for Computational Research grant CNS-0821727.

[1] S. Orlando, G. Sacco, C. Argiroffi, F. Reale, G. Peres, A. Maggio, X-ray emitting mhd accretion shocks in classical t tauri stars. case for moderate to high plasma-beta values, Astronomy and Astrophysics 510 (2010) A71-A82.

[2] D. F. Gray, The Observation and Analysis of Stellar Photospheres, 3rd Edition, Cambridge University Press, Cambridge, United Kingdom, 2008.

[3] D. Odstrcil, V. J. Pizzo, C. N. Arge, Propagation of the 12 may 1997 interplanetary coronal mass ejection in evolving solar wind structures, Journal of Geophysical Research 110 (2005) 2106-2120.

[4] R. Lionello, J. A. Linker, Z. Mikic, Multispectral emission of the sun during the first whole sun month: magnetohydrodynamic simulations, Astrophysical Journal 690 (2009) 902-912.

[5] B. van der Holst, I. V. Sokolov, X. Meng, M. Jin, W. B. Manchester, G. Toth, T. I. Gombosi, Alfven wave solar model (awsom): Coronal heating, Astrophysical Journal 782 (2014) 81-95.

[6] H. Q. Song, J. Zhang, Y. Chen, X. Cheng, Direct observations of magnetic flux rope formation during a solar coronal mass ejection, Astrophysical Journal Letters 792 (2014) L40-L45.

[7] S. Chapman, V. C. A. Ferraro, A new theory of magnetic storms, Nature 126 (1930) 129-130.

[8] J. Raeder, J. Berchem, M. Ashour-Abdalla, L. A. Frank, W. R. Paterson, K. L. Ackerson, S. Kokubun, T. Yamamoto, J. A. Slavin, Boundary layer formation in the magnetotail: Geotail observations and comparisons with a global mhd simulation, Geophysical Research Letters 24 (1997) 951954.

[9] J. G. Lyon, J. A. Fedder, C. M. Mobarry, The lyon-fedder-mobarry (1fm) global mhd magnetospheric simulation code, Journal of Atmospheric and Solar-Terrestrial Physics 66 (2004) 1333-1350. 
[10] G. Toth, B. van der Holst, I. V. Sokolov, D. L. de Zeeuw, T. I. Gombosi, F. Fang, W. B. Manchester, X. Meng, N. Dalal, K. G. Powell, Q. F. Stout, Y. J. Ma, M. Opher, Adaptive numerical algorithms in space weather modeling, Journal of Computational Physics 231 (2012) 870-903.

[11] S. Chapman, V. C. A. Ferraro, A new theory of magnetic storms i.1-5 the initial phase, Terrestrial Magnetism and Atmospheric Electricity 36 (1931) 77-97.

[12] S. Chapman, V. C. A. Ferraro, A new theory of magnetic storms i.6-7 the initial phase 36 (1931) 171-186.

[13] S. Chapman, V. C. A. Ferraro, A new theory of magnetic storms i.8 the initial phase, Terrestrial Magnetism and Atmospheric Electricity 37 (1932) 147-156.

[14] S. Chapman, V. C. A. Ferraro, A new theory of magnetic storms i.9 the initial phase, Terrestrial Magnetism and Atmospheric Electricity 37 (1932) 421-430.

[15] S. Chapman, V. C. A. Ferraro, A new theory of magnetic storms ii.10 the main phase, Terrestrial Magnetism and Atmospheric Electricity 38 (1933) $78-96$.

[16] C. T. Russell, On the relative locations of the bow shocks of the terrestrial planets, Geophysical Review Letters 4 (1977) 387-390.

[17] G. L. Siscoe, Planetary magnetospheres, Reviews of Geophysics and Space Physics 17 (1979) 1677-1693.

[18] C. T. Russell, Planetary magnetism, Reviews of Geophysics and Space Physics 18 (1980) 77-106.

[19] H. C. Zhuang, C. T. Russell, An analytic treatment of the structure of the bow shock and magnetosheath, Journal of Geophysical Research 86 (1981) 2191-2205.

[20] Experimental platform for magnetized hedp science at omega, in: APS Meeting Abstracts, 2012, p. 8073P.

[21] A. J. Cunningham, A. Frank, P. Varniere, S. Mitran, T. W. Jones, Simulating magnetohydrodynamical flow with constrained transport and adaptive mesh refinement: Algorithms and tests of the astrobear code, Astrophysical Journal Supplements 182 (2009) 519-542.

[22] Shot operations 6 target diagnostics gated optical imager (goi), http: //www.lle.rochester.edu/04_resources/04_07_PDM/pdmdocs/ release/D/D-ES/D-ES-P-764/A/D-ES-P-764_REVA.PDF

[23] G. B. Rybicki, A. P. Lightman, Radiative Processes in Astrophysics, 1st Edition, Wiley-Interscience, New York, New York, 1979.

[24] H. K. Chung, W. L. Morgan, R. W. Lee, Flychk: An extension to the kshell spectroscopy kinetics model fly, Journal of Quantum Spectroscopy and Radiation Transfer 81 (2003) 107-115. 